\title{
No Escape-A Novel Tool to Quantify Platelet Intra-Thrombus Movements
}

\author{
Markus Bender ${ }^{1}$ Oliver Otto ${ }^{2}$ \\ ${ }^{1}$ Institute of Experimental Biomedicine I, University Hospital of \\ Würzburg, Würzburg, Germany \\ ${ }^{2}$ ZIK HIKE, University of Greifswald, Greifswald, Germany \\ Thromb Haemost 2018;118:1515-1516.
}

\author{
Address for correspondence Markus Bender, PhD, Institute of \\ Experimental Biomedicine I, University Hospital of Würzburg, \\ Josef-Schneider-Str. 2, D15, 97080 Würzburg, Germany \\ (e-mail: Bender_M1@ukw.de).
}

Platelets are essential players in thrombosis and haemostasis, as they 'survey' the integrity of the vascular system. After vascular injury, platelets are recruited to the exposed subendothelial extracellular matrix that triggers platelet activation to seal wound sites by the formation of a haemostatic plug, thereby preventing excessive bleeding. However, if thrombus formation is uncontrolled under pathological conditions, it may lead to irreversible vessel occlusion and to lifethreatening acute ischaemic disease states, such as myocardial infarction or stroke. ${ }^{1}$

Platelet activation and subsequent thrombus growth involve receptor-ligand interactions, signaling molecules and cytoskeletal proteins which regulate the cellular mechanotransduction. In vitro flow chamber systems combined with whole blood from genetically modified mice or incubated with inhibitors have significantly contributed to a better understanding of the function of platelet proteins in the thrombotic process under shear conditions. Most researchers have analysed their generated real-time movies or off-line images semi-automatically or manually for surface coverage by platelet thrombi (83\%), thrombus volume (78\%) and the number of adhered platelets (56\%). ${ }^{2}$ However, while this method including data evaluation has been used primarily to characterize the role of platelet proteins in thrombus formation, the mechanobiology of platelets in this complex environment had been addressed and understood insufficiently.

In this issue, Tunströmer et al introduce and take advantage of a new computational tool which enables quantitative analysis of platelet intra-thrombus movements. ${ }^{3}$ For this purpose, the authors further developed their in-house image processing script, which has enabled quantification of positional information of individual platelets within a thrombus so $\mathrm{far}^{4}$ They used the acquired positional information of each platelet to track thousands of platelets in a thrombus at the same time and to measure thrombus contraction. ${ }^{3}$

For this, $5 \%$ of platelets were labelled with a platelet-specific antibody and annexin $\mathrm{V}$ was added to whole blood to measure pro-coagulant, phosphatidylserine positive platelets. The samples were either left untreated or incubated with blocking antibodies against glycoprotein (GP) IIb/IIIa and GPIb $\alpha$, or inhibitors blocking P2Y12 or thromboxane production. Then, blood was perfused in a custom-made flow chamber at $400 \mathrm{~s}-1$ over a collagen strip. The authors were able to show how the panel of inhibitors affected thrombus architecture, platelet intra-thrombus movements and thrombus contraction. ${ }^{3}$ More specifically, total platelet accumulation was decreased but the ratio of pro-coagulant platelets to total platelet numbers increased in the presence of inhibitors, particularly when the receptors GPIIb/IIIa and GPIb $\alpha$ were blocked. Platelet movements were dramatically shorter for all samples incubated with inhibitors in contrast to control sample. Furthermore, the authors investigated whether the relative platelet position in a thrombus is a determinant of platelet movement. Platelet movement trajectories were longer and velocity was increased on the outer edge of the thrombus compared with platelets in the thrombus core.

Interestingly, a support vector machine regression approach revealed that initially platelets contracted to small aggregates, which then conjoined to larger aggregates, and finally moved in a homogeneous way towards the centre of the thrombus. The contractile component of platelets strongly contributed to thrombus contraction and was reduced in the presence of inhibitors. Finally, the authors could show that thrombus contraction increased during the first 800 seconds of thrombus formation. ${ }^{3}$

Overall, this big set of data supports and conclusively explains the spatial and functional heterogeneity of a thrombus $^{5,6}$ with respect to the contractile properties. ${ }^{3}$ This advanced analysis tool will be powerful to obtain observerindependent information of thousands of platelets within a

(c) 2018 Georg Thieme Verlag KG Stuttgart · New York
DOI https://doi.org/ $10.1055 / \mathrm{s}-0038-1668587$. ISSN 0340-6245. 
thrombus and to better understand how individual platelets shape thrombus architecture.

Future studies will show whether this unique analysis tool is also suitable for the tracking of other platelet sub-populations, for blood with altered platelet count and size and whether it can be easily used by other researchers. However, knowledge in programming seems to be required to adapt this method to each user.

\section{Funding}

This work was supported by the Deutsche Forschungsgemeinschaft (BE5084/3-1 to M.B. and CRC/TR240 A06 to M. B. and O.O) and the Bundesministerium für Bildung und Forschung (ZIK grant under grant agreement 03Z22CN11 to 0.O.). The authors are solely responsible for the content of this publication.

\section{Conflict of Interest}

None.

\section{References}

1 Nieswandt B, Pleines I, Bender M. Platelet adhesion and activation mechanisms in arterial thrombosis and ischaemic stroke. J Thromb Haemost 2011;9(Suppl 1):92-104

2 Roest M, Reininger A, Zwaginga JJ, King MR, Heemskerk JW; Biorheology Subcommittee of the SSC of the ISTH. Flow chamberbased assays to measure thrombus formation in vitro: requirements for standardization. J Thromb Haemost 2011;9(11): 2322-2324

3 Tunströmer K, Faxälv L, Boknäs N, et al. Quantification of platelet contractile movements during thrombus formation. Thromb Haemost 2018. Doi: 10.1055/s-0038-1668151

4 Claesson K, Lindahl TL, Faxälv L. Counting the platelets: a robust and sensitive quantification method for thrombus formation. Thromb Haemost 2016;115(06):1178-1190

5 Munnix IC, Cosemans JM, Auger JM, Heemskerk JW. Platelet response heterogeneity in thrombus formation. Thromb Haemost 2009;102(06):1149-1156

6 Stalker TJ, Traxler EA, Wu J, et al. Hierarchical organization in the hemostatic response and its relationship to the platelet-signaling network. Blood 2013;121(10):1875-1885 